\title{
VWAP execution as an optimal strategy
}

\author{
Takashi Kato ${ }^{1}$ \\ ${ }^{1}$ Graduate School of Engineering Science, Osaka University, 1-3 Machikaneyama, Toyonaka, \\ Osaka 560-8531, Japan \\ E-mailkato@sigmath.es.osaka-u.ac.jp
}

Received September 7, 2014, Accepted February 23, 2015

\begin{abstract}
The volume weighted average price (VWAP) execution strategy is well known and widely used in practice. In this study, we explicitly introduce a trading volume process into the AlmgrenChriss model, which is a standard model for optimal execution. We then show that the VWAP strategy is the optimal execution strategy for a risk-neutral trader. Moreover, we examine the case of a risk-averse trader and derive the first-order asymptotic expansion of the optimal strategy for a mean-variance optimization problem.
\end{abstract}

Keywords optimal execution problem, trading volume, volume weighted average price (VWAP), market impact

Research Activity Group Mathematical Finance

\section{Introduction}

Recently, researchers in the field of mathematical finance have increasingly begun studying the optimal execution problem. Bertsimas and Lo [1] and Almgren and Chriss [2] are the classic and standard studies in the field, while Gatheral and Schied [3] provide a survey of dynamic models that address execution problems.

When studying execution problems, we should take care of the market impact (MI), which is the effect that a trader's investment behavior has on security prices. As pointed out by [4], the price recovery effect is efficient as another property that affects the trader's execution schedule. The price recovery effect is also recognized as the resilience of the MI or transient MI, and several studies have proposed optimal execution models with MI functions and resilience functions (see [3] and the references therein).

Furthermore, trading volume (turnover) is another important factor in execution problems. Trading volume is a representative index of financial market activity. If trading volume is high, the security is highly liquid, and a trader can easily liquidate shares of the security. As an execution strategy that exploits trading volume, the volume weighted average price (VWAP) strategy is well known and widely used in practice [5]. The execution speed of the VWAP strategy is proportional to the trading volume of the relevant security. Although the VWAP strategy is a standard execution strategy, it remains unclear why it is effective in terms of optimal execution theory.

The purpose of this study is to investigate whether the VWAP strategy is in fact optimal in an execution problem equipped with explicitly defined trading volume processes. Here, we introduce the generalized AlmgrenChriss (AC) model, in which the temporary MI function is affected by the trading volume. Then, we show that the VWAP strategy is optimal when the trader is risk neutral. Moreover, we study the case of a risk-averse trader and present the second-order linear ordinal differential equation (ODE), in which the solution is the optimal strategy of the corresponding mean-variance optimization problem. Finally, we also provide the first-order asymptotic expansion for the optimal strategy.

\section{VWAP and VWAP strategies}

In this section, we briefly introduce VWAP and define VWAP execution strategies. First, $S_{t}$ denotes a security price at time $t \in[0, T]$, where $T>0$ is a time horizon. Mathematically, $\left(S_{t}\right)_{t}$ is regarded as a continuous-time stochastic process. The strict definition of $\left(S_{t}\right)_{t}$ is omitted in this section, but is provided in the next section.

Then, $V_{t}$ denotes a cumulative trading volume process on the time interval $[0, T]$. We assume that $V_{t}$ is continuously differentiable and strictly increasing. That is, there is a positive continuous process $\left(v_{t}\right)_{t}$, such that

$$
V_{t}=\int_{0}^{t} v_{r} d r, \quad t \in[0, T], \quad \text { a.s. }
$$

Then, the VWAP of the security at $t=T$ is defined as

$$
S_{T}^{\mathrm{VWAP}}=\frac{\int_{0}^{T} S_{t} v_{t} d t}{\int_{0}^{T} v_{t} d t}=\frac{1}{V_{T}} \int_{0}^{T} S_{t} v_{t} d t .
$$

Next, we define the trader's own VWAP. We consider a single trader who has a large number of shares, $\Phi>0$, at initial time $t=0$, which he/she tries to liquidate until $t=T$. A trader's execution strategy is denoted by $\boldsymbol{\zeta}=\left(\zeta_{t}\right)_{t}$, where $\zeta_{t} \geq 0$ is the execution (selling) speed at time $t$. We assume the following "sell-off condition" [6]:

$$
\int_{0}^{T} \zeta_{t} d t=\Phi
$$

This condition implies that the trader must sell all securities held until the time horizon. The trader's VWAP is 
defined by

$$
S_{T}^{\mathrm{vwap}}(\boldsymbol{\zeta})=\frac{\int_{0}^{T} S_{t} \zeta_{t} d t}{\int_{0}^{T} \zeta_{t} d t} .
$$

Then, the VWAP execution strategy are defined as follows.

Definition $1 \quad \zeta=\left(\zeta_{t}\right)_{t}$ is called a VWAP strategy if $\zeta_{t}=\gamma v_{t}, t \in[0, T]$ a.s., for some $\gamma>0$.

Note that $\gamma$ is called an involvement ratio. It is immediately shown that if $\boldsymbol{\zeta}$ is a VWAP strategy, then $S_{T}^{\text {vwap }}(\boldsymbol{\zeta})$ coincides with $S_{T}^{\mathrm{VWAP}}$ a.s. To sell $\Phi$ shares of the security using the VWAP strategy, $\gamma$ is set so that $\boldsymbol{\zeta}$ satisfies (3). That is,

$$
\Phi=\int_{0}^{T} \gamma v_{t} d t=\gamma V_{T}
$$

which implies that $\gamma=\Phi / V_{T}$. However, such a strategy cannot be executed in practice, because the value of $V_{T}$ is unobservable before the time horizon, $T$.

The difference between two VWAPs, $S_{T}^{\mathrm{vwap}}(\boldsymbol{\zeta})$ and $S_{T}^{\text {VWAP }}$, is called a VWAP slippage. Minimizing VWAP slippage problems is studied by $[7,8]$.

\section{The AC model with trading volume}

In this section, we introduce our model of an optimal execution problem. Our model is based on the AC model, as proposed by [2] and generalized by the authors of [3].

Let $\left(\Omega, \mathcal{F},\left(\mathcal{F}_{t}\right)_{t}, P\right)$ be a stochastic basis and let $\left(S_{t}^{0}\right)_{t}$ be an $\left(\mathcal{F}_{t}\right)_{t}$-martingale satisfying

$$
\mathrm{E}\left[\left(S_{T}^{0}\right)^{2}\right]<\infty
$$

Here, $S_{t}^{0}$ is regarded as an unaffected price of the security at time $t$. In other words, it is the security price when there is no MI.

The execution strategy $\boldsymbol{\zeta}=\left(\zeta_{t}\right)_{t}$ is referred to as admissible if $\boldsymbol{\zeta}=\left(\zeta_{t}\right)_{t}$ is $\left(\mathcal{F}_{t}\right)_{t}$-adapted and satisfies the sell-off condition (3). The set of admissible strategies is denoted by $\mathcal{A}(\Phi)$. Under the given admissible strategy, $\zeta \in \mathcal{A}(\Phi)$, the security price, $S_{t}$, is defined as follows:

$$
S_{t}=S_{t}^{0}-\int_{0}^{t} g\left(\zeta_{r}\right) d r-\tilde{g}\left(v_{t}, \zeta_{t}\right),
$$

where $g$ (resp., $\tilde{g}$ ) is a permanent (resp., temporary) MI function and $\left(v_{t}\right)_{t}$ is a positive $\left(\mathcal{F}_{t}\right)_{t}$-adapted process describing the instantaneous trading volume.

In this study, $g$ is always assumed to be a linear function:

$$
g(\zeta)=\kappa \zeta
$$

for some $\kappa>0$. This assumption is necessary given the view of an absence of price manipulation. See [3] for more detail.

The temporary MI function, $\tilde{g}$, depends on the execution strategy, $\zeta_{t}$, and the trading volume, $v_{t}$. It is natural that the temporary MI is decreasing as the trading volume increases, because a large trading volume implies high market liquidity. Therefore, we adopt the following form as the function $\tilde{g}$ :

$$
\tilde{g}(v, \zeta)=\frac{\tilde{\kappa} \zeta}{v},
$$

where $\tilde{\kappa}>0$.

Remark 2 We can generalize the form of $\tilde{g}$ as

$$
\tilde{g}(v, \zeta)=k(v) \zeta^{\alpha},
$$

where $\alpha>0$ and $k$ is a positive continuous function. See also Remark 5 below.

Next, we define our objective function. For a given $\boldsymbol{\zeta}$, an implementation shortfall (IS) cost is defined as

$$
\mathcal{C}(\boldsymbol{\zeta})=S_{0} \Phi-\int_{0}^{T} S_{t} \zeta_{t} d t .
$$

Substituting (4) and applying the integration by parts formula, we can rewrite (7) as

$$
\mathcal{C}(\boldsymbol{\zeta})=\frac{\kappa \Phi^{2}}{2}-\int_{0}^{T} \varphi_{t} d S_{t}^{0}+\tilde{\kappa} \int_{0}^{T} \frac{\zeta_{t}^{2}}{v_{t}} d t
$$

where

$$
\varphi_{t}=\Phi-\int_{0}^{t} \zeta_{r} d r
$$

denotes the remaining shares of the security held at time $t$.

We define the following three classes of admissible strategies:

$$
\begin{aligned}
& \mathcal{A}^{\text {ant }}(\Phi)=\left\{\boldsymbol{\zeta} \in \mathcal{A}(\Phi) ; \mathcal{G}_{T} \text {-measurable }\right\}, \\
& \mathcal{A}^{\text {adap }}(\Phi)=\left\{\boldsymbol{\zeta} \in \mathcal{A}(\Phi) ;\left(\mathcal{G}_{t}\right)_{t} \text {-adapted }\right\}, \\
& \mathcal{A}^{\text {stat }}(\Phi)=\left\{\boldsymbol{\zeta} \in \mathcal{A}(\Phi) ; \text { independent of }\left(\mathcal{G}_{t}\right)_{t}\right\},
\end{aligned}
$$

where $\left(\mathcal{G}_{t}\right)_{t}$ is a filtration generated by $\left(v_{t}\right)_{t}$.

Strategy $\zeta \in \mathcal{A}^{\text {ant }}(\Phi)$ is called an anticipating strategy. In this case, the trader knows the value of $V_{T}$ (the cumulative trading volume defined by (1)) at the initial time. Therefore, he/she can execute the VWAP strategy with the involvement ratio $\Phi / V_{T}$. Note that in this case it should hold that $\mathcal{G}_{T} \subset \mathcal{F}_{0}$. This case is unrealistic, but the optimal anticipating strategy gives us a benchmark for execution strategies.

Strategy $\boldsymbol{\zeta} \in \mathcal{A}^{\text {adap }}(\Phi)$ is called an adaptive strategy. It is natural to search for an optimal strategy from within the adaptive strategies, but the problem may become difficult.

Strategy $\zeta \in \mathcal{A}^{\text {stat }}(\Phi)$ is called a static strategy. In trading practice, a VWAP strategy is often classified as a static (deterministic) strategy. In this case, an estimated cumulative trading volume is used as a substitute for $V_{T}$.

\subsection{Risk-neutral case}

In this subsection, we examine the case in which the trader is risk neutral. Our purpose is to minimize the expected IS cost,

$$
\mathrm{E}[\mathcal{C}(\boldsymbol{\zeta})]=\frac{\kappa \Phi^{2}}{2}+\tilde{\kappa} \mathrm{E}\left[\int_{0}^{T} \frac{\zeta_{t}^{2}}{v_{t}} d t\right] .
$$

Here, equality (9) follows from the martingale property of $\left(S_{t}^{0}\right)_{t}$. We can then show the following two theo- 
rems by applying the Jensen inequality and appropriate changes of variables.

Theorem $3 \quad \hat{\zeta}_{t}=v_{t} \Phi / V_{T}$ is the optimal anticipating strategy for the problem $\inf _{\boldsymbol{\zeta} \in \mathcal{A}^{\text {ant }}(\Phi)} \mathrm{E}[\mathcal{C}(\boldsymbol{\zeta})]$.

Theorem 4 Set $u_{t}=1 / \mathrm{E}\left[1 / v_{t}\right]$ and assume that $u_{t}$ is finite and continuous in $t$. Then, $\tilde{\zeta}_{t}=u_{t} \Phi / U_{T}$ is the optimal static strategy for the problem $\inf _{\boldsymbol{\zeta} \in \mathcal{A}^{\text {stat }}(\Phi)} \mathrm{E}[\mathcal{C}(\boldsymbol{\zeta})]$, where $U_{T}=\int_{0}^{T} u_{t} d t$.

The strategy $\left(\hat{\zeta}_{t}\right)_{t}$ is the VWAP strategy. Therefore, Theorem 3 gives the typical case in which the VWAP strategy is optimal for the execution problem. Note again that the "exact" VWAP strategy, $\left(\hat{\zeta}_{t}\right)_{t}$, is not executable without observing the future value of the cumulative trading volume, $V_{T}$. However, it provides a benchmark strategy for the optimal execution problem.

Theorem 4 tells us how to mimic the VWAP strategy, $\left(\hat{\zeta}_{t}\right)_{t}$. The expected VWAP strategy $\left(\tilde{\zeta}_{t}\right)_{t}$ is optimal in the class of static strategies, and the expectation $u_{t}$ of $v_{t}$ is calculated in the sense of a harmonic mean. In practice, the estimated value of the trading volume based on historical data is often applied to replicate the VWAP strategy. The assertion of Theorem 4 is consistent with such a situation.

Remark 5 We can also show the above two theorems when $\tilde{g}$ has a more general form, as in (6). In this case, $\hat{\zeta}_{t}$ is given as $\hat{\zeta}_{t}=\tilde{v}_{t} \Phi / \tilde{V}_{T}$, where

$$
\tilde{v}_{t}=\frac{1}{k\left(v_{t}\right)^{1 / \alpha}}, \quad \tilde{V}_{T}=\int_{0}^{T} \tilde{v}_{t} d t .
$$

We refer to $\left(\hat{\zeta}_{t}\right)_{t}$ as a "twisted" VWAP strategy. Similarly, $\tilde{\zeta}_{t}$ is changed to $\tilde{\zeta}_{t}=\tilde{u}_{t} \Phi / \tilde{U}_{T}$, where

$$
\tilde{u}_{t}=\frac{1}{\mathrm{E}\left[k\left(v_{t}\right)\right]^{1 / \alpha}}, \quad \tilde{U}_{T}=\int_{0}^{T} \tilde{u}_{t} d t .
$$

Finally, we consider the adaptive case. Here, we only consider the special case in which $\left(v_{t}\right)_{t}$ follows a geometric Brownian motion:

$$
d v_{t}=v_{t}\left(\mu d t+\sigma d B_{t}\right), \quad t \geq 0, \quad v_{0}>0,
$$

where $\mu \in \mathbb{R}, \sigma>0$, and $\left(B_{t}\right)_{t}$ is a one-dimensional $\left(\mathcal{F}_{t}\right)_{t}$-Brownian motion. Then, we obtain the following theorem using a standard verification argument of dynamic control theory.

Theorem 6 Assume $\mathrm{E}\left[U_{T}\right]<\infty$. Then $\tilde{\zeta}_{t}$, defined in Theorem 4 , is the optimal adaptive strategy for the problem $\inf _{\boldsymbol{\zeta} \in \mathcal{A}^{\text {adap }(\Phi)}} \mathrm{E}[\mathcal{C}(\boldsymbol{\zeta})]$.

This theorem implies that the optimal adaptive strategy coincides with the expected VWAP strategy, which is also optimal among the static strategies.

Remark 7 When $v_{t}$ is a constant, the optimal strategy is selling with constant speed (i.e., $\left.\hat{\zeta}_{t}=\tilde{\zeta}_{t}=\Phi / T\right)$. This strategy is called a time weighted average price (TWAP) strategy. In $[6,9]$, we find similar examples such that the TWAP strategy is the optimal strategy for a risk-neutral trader when the permanent $M I$ function is non-linear. In particular, [9, Theorem 7.4] gives the example of an " $S$ shaped" MI function, which is often observed in practice.

\subsection{Mean-variance optimization}

Next, we study the following static mean-variance optimization problem:

$$
\inf _{\boldsymbol{\zeta} \in \mathcal{A}^{\text {stat }}(\Phi)} \operatorname{MV}^{\lambda}(\mathcal{C}(\boldsymbol{\zeta}))
$$

where $\lambda \geq 0$ and

$$
\operatorname{MV}^{\lambda}(\mathcal{C}(\boldsymbol{\zeta}))=\mathrm{E}[\mathcal{C}(\boldsymbol{\zeta})]+\lambda \operatorname{Var}(\mathcal{C}(\boldsymbol{\zeta}))
$$

The parameter $\lambda$ denotes the measure of the trader's risk aversion. When $\lambda=0$, the above problem is equivalent to that of the previous subsection.

In this subsection, we assume the following:

- $S_{t}^{0}=S_{0}^{0}+\tilde{\sigma} \tilde{B}_{t}$, where $\tilde{\sigma}>0$ and $\left(\tilde{B}_{t}\right)_{t}$ is a one dimensional $\left(\mathcal{F}_{t}\right)_{t}$-Brownian motion.

- $v_{t}$ is deterministic, $v_{t} \in C^{1}((0, T))$ and $v_{t} \geq \delta, t \in$ $[0, T]$ for some $\delta>0$ ( $v_{0}$ and $v_{T}$ may diverge).

By a straightforward calculation, we see that

$$
\operatorname{MV}^{\lambda}(\mathcal{C}(\boldsymbol{\zeta}))=\frac{\kappa \Phi^{2}}{2}+\tilde{\kappa} f^{\lambda}(\Phi ; \boldsymbol{\zeta})
$$

for $\zeta \in \mathcal{A}^{\text {stat }}(\Phi)$, where

$$
f^{\lambda}(\Phi ; \boldsymbol{\zeta})=\int_{0}^{T}\left\{\frac{\tilde{\sigma}^{2} \lambda}{\tilde{\kappa}} \varphi_{t}^{2}+\frac{\zeta_{t}^{2}}{v_{t}}\right\} d t
$$

and $\left(\varphi_{t}\right)_{t}$ is defined as in (8). Therefore, our optimization problem is equivalent to the following variational problem:

$$
\inf _{\varphi} \int_{0}^{T} F\left(t, \varphi_{t}, \dot{\varphi}_{t}\right) d t
$$

subject to $\varphi_{0}=\Phi$ and $\varphi_{T}=0$, where $\varphi=\left(\varphi_{t}\right)_{t \in[0, T]}$ is absolutely continuous and

$$
F(t, \varphi, \zeta)=\frac{\tilde{\sigma}^{2} \lambda}{\tilde{\kappa}} \varphi^{2}+\frac{\zeta^{2}}{v_{t}}
$$

From the standard theory of variational analysis, we can show the following theorem.

Theorem 8 There is a unique optimizer to (11) satisfying the following second-order linear ODE with variable coefficients:

$$
\ddot{\varphi}_{t}-a_{t} \dot{\varphi}_{t}-\frac{\tilde{\sigma}^{2} \lambda}{\tilde{\kappa}} \varphi_{t}=0
$$

where

$$
a_{t}=\frac{\dot{v}_{t}}{v_{t}}=\frac{d}{d t} \log v_{t} .
$$

The above theorem implies that we can find the optimizer by solving the ODE (12) with boundary conditions $\varphi_{0}=\Phi$ and $\varphi_{T}=0$, and the optimal strategy of the problem (10) is obtained as $\zeta_{t}=-\dot{\varphi}_{t}$.

Remark 9 If $v_{t}$ is a constant, the optimal strategy is explicitly obtained in [2] as

$$
\zeta_{t}^{\lambda}=\frac{\cosh \left(\gamma_{\lambda}(T-t)\right)}{\sinh \left(\gamma_{\lambda} T\right)} \gamma_{\lambda} \Phi,
$$

where $\gamma_{\lambda}=\tilde{\sigma}^{2} \lambda / \tilde{\kappa}$. The corresponding process, $\left(\varphi_{t}\right)_{t}$, is given by

$$
\frac{\sinh \left(\gamma_{\lambda}(T-t)\right)}{\sinh \left(\gamma_{\lambda} T\right)} \Phi
$$


In this case, (12) becomes the ODE with constant coefficients. Then the solution can be explicitly solved and coincides with (13).

Here, we formally derive the first-order asymptotic expansion of the optimal strategy of (10) with small $\lambda$. Let $\boldsymbol{\zeta}^{\lambda}=\left(\zeta_{t}^{\lambda}\right)_{t}$ be the optimal strategy of (10) and put $\varphi_{t}^{\lambda}=\Phi-\int_{0}^{t} \zeta_{r}^{\lambda} d r$. We already know that when $\lambda=0$, it holds that

$$
\zeta_{t}^{0}=\frac{v_{t} \Phi}{V_{T}}, \quad \varphi_{t}^{0}=\left(1-\frac{V_{t}}{V_{T}}\right) \Phi .
$$

Define $\tilde{\varphi}_{t}^{\lambda}=\left(\varphi_{t}^{\lambda}-\varphi_{t}^{0}\right) / \lambda$. Then we can easily check that $\left(\tilde{\varphi}_{t}^{\lambda}\right)_{t}$ is a solution to

$$
\ddot{\tilde{\varphi}}_{t}^{\lambda}-a_{t} \dot{\tilde{\varphi}}_{t}^{\lambda}-v_{t}\left(\varphi_{t}^{0}+\frac{\tilde{\sigma}^{2} \lambda}{\tilde{\kappa}} \tilde{\varphi}_{t}^{\lambda}\right)=0
$$

with boundary conditions $\tilde{\varphi}_{0}^{\lambda}=\tilde{\varphi}_{T}^{\lambda}=0$. Letting $\lambda \rightarrow 0$, we get

$$
\ddot{\tilde{\varphi}}_{t}^{0}-a_{t} \dot{\tilde{\varphi}}_{t}^{0}=v_{t}\left(1-\frac{V_{t}}{V_{T}}\right) \Phi
$$

Hence,

$$
\tilde{\varphi}_{t}^{0}=\Phi\left\{t V_{t}-\left(1+\frac{V_{t}}{V_{T}}\right) \mathcal{V}_{t}-\frac{1}{V_{T}} \int_{0}^{t} V_{r}^{2} d r+C V_{t}\right\},
$$

where

$$
C=\frac{2 \mathcal{V}_{T}}{V_{T}}-T-\frac{1}{V_{T}^{2}} \int_{0}^{T} V_{r}^{2} d r, \quad \mathcal{V}_{t}=\int_{0}^{t} V_{r} d r
$$

Now we get the following asymptotic expansion formula around the VWAP strategy:

$$
\zeta_{t}^{\lambda}=\frac{v_{t} \Phi}{V_{T}}+\lambda \tilde{\zeta}_{t}^{0}+o(\lambda), \quad \lambda \rightarrow 0
$$

where $\tilde{\zeta}_{t}^{0}=-\dot{\tilde{\varphi}}_{t}$ is given by

$\tilde{\zeta}_{t}^{0}=\Phi v_{t}\left\{T-t-\frac{\mathcal{V}_{T}-\mathcal{V}_{t}}{V_{T}}-\frac{1}{V_{T}}\left(\mathcal{V}_{T}-\int_{0}^{T} V_{r}^{2} d r\right)\right\}$.

Remark 10 In fact, we can also solve (10) numerically (even when $\left(v_{t}\right)_{t}$ is not deterministic) by, for instance, using the sequential quadratic programming algorithm.

Finally, we give a numerical example of an "arcsine cumulative trading volume process." For brevity, set $T=1$. Assume that $v_{t}=1 /(\pi \sqrt{t(1-t)})$. Then $V_{t}$ is given by the arcsine function: $V_{t}=2 \pi^{-1} \arcsin \sqrt{t}$. Note that $v_{t} \longrightarrow \infty$ as $t \rightarrow 0$ and $t \rightarrow 1$ : this reflects the realistic situation in which the market is active near the opening and closing, but becomes less active in the continuous session. Then, we investigate the optimal strategies when setting $\lambda=0$ (VWAP), $0.5,1$, and 2. The other parameters are set as $\tilde{\sigma}=0.1, \tilde{\kappa}=0.02$, and $\Phi=1$. The results are shown in Fig. 1. Here, we find that the execution speed with small $t$ becomes larger when $\lambda$ is large. This is because a large $\lambda$ implies that the trader is risk averse and wants to quickly liquidate the security to avoid volatility risk.

\section{Concluding remarks}

In this study, we studied the generalized AC model while considering trading volume. Here, we showed that,

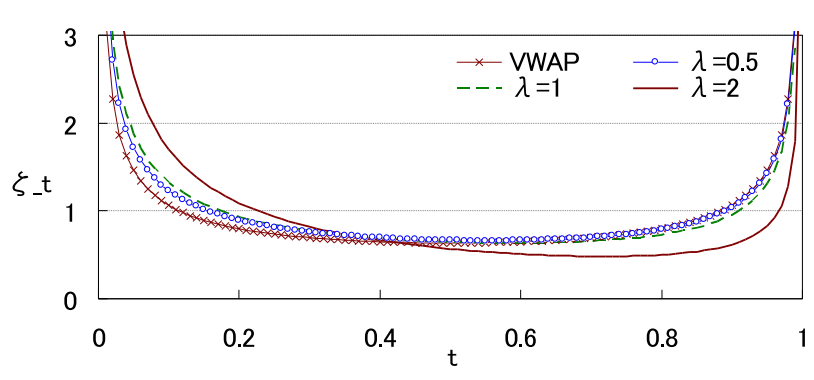

Fig. 1. The forms of optimal strategies $\left(\zeta_{t}\right)_{t}$ of (10) with the arcsine cumulative trading volume process; the horizontal axis shows time, $t$; the vertical axis is $\zeta_{t}$.

with some standard settings, the VWAP execution strategy is the optimal strategy for a risk-neutral trader. When considering adaptive strategies, we obtained our result only when the trading volume process follows a geometric Brownian motion. Studying more general cases of adaptive optimization problems is an area of future research.

In this study, we only considered volume-dependent temporary MI functions. Therefore, it remains to study the case in which the permanent MI function depends on the trading volume.

As mentioned in Remark 7, when the trader is risk neutral, there are examples other than the AC-based model in which the TWAP strategy is optimal. Therefore, it would also be interesting to investigate whether the optimal strategy is a VWAP strategy in the model of $[6,9]$ by explicitly introducing trading volume processes so that the time parameter is replaced by "volume time (stochastic clock)."

\section{Acknowledgments}

The author thanks the anonymous referee for careful reading and helpful comments.

\section{References}

[1] D. Bertsimas and A. W. Lo, Optimal control of execution costs, J. Fin. Mark., 1 (1998), 1-50.

[2] R. Almgren and N. Chriss, Optimal execution of portfolio transactions, J. Risk, 18 (2000), 57-62.

[3] J. Gatheral and A. Schied, Dynamical models of market impact and algorithms for order execution, Handbook on Systemic Risk, J. P. Fouque and J. Langsam eds., (2013), 579-602.

[4] T. Kato, An optimal execution problem with a geometric Ornstein-Uhlenbeck price process, preprint.

[5] A. Madhavan, VWAP Strategies, Trading, 1 (2002), 32-39.

[6] T. Kato, An optimal execution problem with market impact, Financ. Stoch., 18 (2014), 695-732.

[7] C. Frei and N. Westray, Optimal execution of a VWAP order: a stochastic control approach, Math. Financ., (2013), DOI: $10.1111 /$ mafi.12048.

[8] H. Konishi, Optimal slice of a VWAP trade, J. Finan. Mark., 5 (2002), 197-221.

[9] T. Kato, Non-linearity of market impact functions: empirical and simulation-based studies on convex/concave market impact functions and derivation of an optimal execution model (in Japanese), Trans. JSIAM, 24 (2014), 203-237. 